\title{
Comprender la economía: Educación económica y financiera en la infancia desde una perspectiva constructivista
}

\author{
Marianela Denegri Coria ${ }^{1}$ \\ Jocelyne Sepúlveda Aravena ${ }^{2}$ \\ Francisca Silva Layera ${ }^{3}$
}

\begin{abstract}
RESUMO
La globalización y los cambios estructurales a nivel económico, social y político han modificado las dinámicas relacionales en la sociedad y han otorgado un papel protagónico al consumo (BAUMANN, 2007), situación transversal a todas las edades. Un segmento especialmente relevante son los niños, quienes enfrentan las demandas del medio social y vivencian la construcción de su identidad en un entorno de alta mercantilización. En este artículo se aborda la descripción y análisis de los niveles de compresión económica desde la infancia y los actores y procesos que configuran la socialización económica, definida como un proceso de aprendizaje de pautas de interacción con el mundo económico (DENEGRI et al., 2004). A partir de un enfoque constructivista piagetiano, se propone un modelo de educación económica y financiera que se articula con los requerimientos de la formación ciudadana. Se concluye con reflexiones en torno a la importancia de la educación económica y financiera para el desarrollo de una ciudadanía crítica y económicamente empoderada desde la infancia.
\end{abstract}

PALAVRAS-CHAVE: Educación económica y financiera, socialización económica, niñez y juventud, ciudadanía.

\footnotetext{
${ }^{1}$ Doctora en Psicología. Centro de Excelencia en Psicología Económica y del Consumo, Universidad de La Frontera, Temuco, Chile.E-mail: marianela.denegri@ufrontera.cl

${ }^{2}$ Magíster en Psicología. Centro de Excelencia en Psicología Económica y del Consumo, Universidad de La Frontera, Temuco, Chile.E-mail: jocelyne.sepulveda@ufrontera.cl

${ }^{3}$ Licenciada en Psicología. Centro de Excelencia en Psicología Económica y del Consumo, Universidad de La Frontera, Temuco, Chile.E-mail: francisca.silva@ufrontera.cl
} 
Understanding economics: economic and financial education in child hood from a constructivist perspective

\begin{abstract}
Globalization and structural changes at the economic, social and political level shave modified the relational dynamics in society and have given a leading role to consumption (BAUMANN, 2007), a situation that transcends all ages. A particular lyre levant segmentist he children, who faces the demands of the social environment and experiences the construction identity in an environment of high com modification. This article deals witht he description and analysis of levels of economic compression from child hood and the actors and processes that shapes the economic socialization, defined as a process of learning patterns of interaction with the economic world (DENEGRI et al., 2004). Based on a Piagetian constructivist approach, a model of economic and financial education is proposed that articulate es with there quire ments of citizen education. It concludes with reflection son the importance of economic and financial education for the development of a critically and economically empowered citizen ship from childhood.
\end{abstract}

KEYWORDS: Economic and financial education, economic socialization, childhood and youth, citizenship.

$$
* * *
$$

\title{
Introdução
}

En Chile, la progresiva globalización y el cambio estructural em función del conocimiento y del intercambio de información, cambió para siempre las dinámicas de relaciones al interior de la sociedad, dando le un poder inusitado a ciertas prácticas, dentro de las cuales destaca de manera particular, el consumo y su valoración (BAUMANN, 2007; DENEGRI; MARTÍNEZ, 2004), no sólo como un medio de satisfacción de aquellas necesidades esenciales o básicas, sino como un referente de definición identitaria tanto a nivel individual como colectivo y una variable que influye fuertemente en La percepción de bien estar subjetivo de la población. 
A su vez se han producido profundos cambios en los valores de la sociedad, la que ha cambiado desde una concepción de austeridad altamente valorada, hacia la búsqueda del hedonismo y la satisfacción inmediata de los deseos, flexibilizando el concepto y actitud hacia La deuda, lo que ha permitido mayor desarrollo económico (mayor consumo), pero al mismo tiempo ha aumentado El riesgo de las operaciones financieras especialmente en las poblaciones más vulnerables como es el caso de los jóvenes, especialmente de los adolescentes, por el impacto que ello puede tener em su futura seguridad financiera (NORVILITIS; SAZBLICKI; WILSON, 2003; NORVILITIS; SANTA MARÍA，2002; LYONS, 2004;AMAR et al., 2003; DENEGRI; MARTÍNEZ; ETCHEBARNE, 2007; LUSARDI; TRUFANO, 2009).

Sin embargo, las modernas sociedades globalizadas no necesariamente entregan a sus ciudadanos las herramientas para comprender La complejidad de los cambios a los que están cotidianamente expuestos. Uno de estos vacíos se vincula a las formas de incorporar el conocimiento económico necesario para comprenderlos profundos cambios que ha sufrido la humanidad como producto de los procesos globalizadores (KHAN; NARU, 2006; LUSARDI; TRUFANO, 2009; LUSARDI; MITCHELL, 2013; BRAUNSTEIN, 2008; LUSARDI; MITCHELL, 2007; LUSARDI; TRUFANO, 2009; ORGANISATION FOR ECONOMIC COOPERATION AND DEVELOPMENT [OECD]，2005a， 2005b，2008，2009,PNUD， 2009, DENEGRI; MARTÍNEZ, 2004; SALEMI, 2005; SANTOMERO, 2003).

En este escenario, resulta crucial apoyar a niños y adolescentes para que comprendan las cuestiones financieras y desarrollen competencias, actitudes y valores que les protejan frente a la presión constante para un consumo poco reflexivo. Esta generación, según lo establecen los análisis de la OECD (2012) deberá soportar muchos más riesgos financieros en la edad adulta que sus padres, especialmente aquellos vinculados a las estrategias de logro de ahorro en un entorno laboral cada vez más inestable, la 
planificación para la jubilación y la necesidad de cubrir sus necesidades personales y familiares de cuidado de la salud.

\section{La comprensión de la economía en la niñez y adolescencia}

A modo de contexto, cerca de 2,5 billones de personas, o un tercio de La población mundial, se espera que sean menores de 18 años para el año 2020 (ZMUDA, 2011). Estos antecedentes permiten comenzar a dimensionar La estrecha vinculación entre economía y niñez/adolescencia.

La mayor parte de las primeras investigaciones que se preguntaron ¿cómo los niños y niñas llegan a comprender el mundo económico? se centraron en la comprensión de los conceptos económicos en la infancia y adolescencia a partir de la perspectiva piagetana del desarrollo cognitivo y comprobaron que en la niñez se construyen activamente explicaciones acerca del mundo económico y que estas explicaciones están a la base de sus conductas de consumo (BERTI; BOMBI, 1988; BERTI; GRIVET, 1990; JAHODA, 1983; FURTH, 1980). Ello nos permitió comprender que las ideas que expresan los niños acerca del mundo social son cualitativamente diferentes de las de los adultos y que el cambio cognitivo, evidenciado en el desarrollo hacia una mayor coherencia y organización interna, expresa un proceso de construcción que no puede ser atribuido solo a la influencia de los conocimientos transmitidos por los adultos, sino a un esfuerzo activo del niño por seleccionar, organizar y hacer coherentes las experiencias e informaciones sociales de acuerdo a las posibilidades de su aparato cognitivo. Esto implica que la comprensión de la economía puede ser interpretada como un proceso de diferenciación y articulación en el cual el niño construye reglas cada vez más precisas y complejas (FURTH, 1980; JAHODA, 1983, 1984; BERTI; BOMBI, 1988; BERTI; GRIVET, 1990).

A partir de un enfoque psicogenético, nuestro equipo de investigación también ha realizado una serie de trabajos sobre comprensión de la economía en la infancia y adolescencia en España, Chile, Colombia y Brasil 
(AMAR; ABELLO; DENEGRI, 2001; ARAUJO, 2009; DENEGRI, 1995a; 1995b; 1997; DENEGRI et al., 1998; DENEGRI et al., 2000). Los resultados obtenidos mostraron la existencia de una secuencia evolutiva caracterizada por un patrón de cambio conceptual en una construcción progresiva en una secuencia evolutiva, en la cual el razonamiento en torno a la economía se complejiza a medida que los sujetos avanzan en edad, por lo que los cambios en las representaciones o explicaciones que los individuos manejan para explicarse la economía, responden a etapas de un proceso constructivo y progresivo mediado por factores como la edad, el contacto con el dinero y las oportunidades de socialización económica (DENEGRI, 1995b, AMAR et al., 2002; Ello nos permitió postular la presencia de un modelo de Psicogénesis del pensamiento económico que se expresa en tres niveles de desarrollo: Nivel I Pensamiento extraeconómico y económico primitivo (correspondientes a los niños de 6 a 9 años), Nivel II Pensamiento económico subordinado (que corresponde a los niños mayores de 10 años, adolescentes e incluso algunos adultos) y Nivel III Pensamiento económico Independiente o inferencial (correspondientes a adolescentes mayores y adultos).

Al someter este modelo a validación empírica, se observan diferencias significativas en la distribución de los sujetos por edad para cada uno de los niveles, apreciándose que los niños de 6 a 10 años tienden a ubicarse mayoritariamente en un nivel de pensamiento económico primitivo, mientras que los sujetos entre 11 a 14 años se ubican en el nivel de pensamiento económico subordinado. Ello corresponde a lo esperable de acuerdo a su manejo de informaciones económicas y a las herramientas cognitivas que disponen. Sin embargo, llama la atención el alto porcentaje de adolescentes entre 15 a 18 años que permanece en un nivel de pensamiento económico subordinado y el escaso porcentaje que alcanza el nivel de pensamiento económico inferencial, que sería el esperable teóricamente para su edad.

Aparecen además diferencias significativas entre niveles socioeconómicos, donde los adolescentes de nivel socioeconómico bajo tienden 
a permanecer mayoritariamente en el nivel de pensamiento económico subordinado en comparación con los individuos del nivel socioeconómico alto que alcanzan el nivel inferencial y, por lo tanto, logran una comprensión más eficiente del sistema económico y del origen y circulación del dinero. Algo similar sucede asociado al lugar de residencia, apreciándose que los adolescentes que proceden de ciudades multifinancieras desarrollan una comprensión más completa y articulada del funcionamiento económico (AMAR; ABELLO; DENEGRI, 2001; ARAUJO, 2009; DENEGRI, 1995a; 1995b; 1997; GEMPP et al., 2006; DENEGRI et al., 1998; DENEGRI et al., 2000; DENEGRI; MARTÍNEZ; ETCHEBARNE, 2007).

Los niños y jóvenes se socializan económica y financieramente no solo en las redes educativas formales sino también de lãs interacciones com los agentes de socialización como amigos, familia y medios de comunicación (HILGERT, HOGARTH y BEVERLY, 2003), siendo los puntos que abordaremos a continuación.

\section{La socialización económica en la infancia: actores y procesos}

La socialización económica es definida como un proceso de aprendizaje de pautas de interacción con el mundo económico mediante La construcción activa por parte del niño, de conocimientos, destrezas, estrategias, patrones de comportamiento y actitudes acerca del uso del dinero y su valor en La sociedad. En este proceso intervienen varios agentes de socialización, siendo la familia el primero de ellos y probablemente uno de los más importantes (DENEGRI, PALAVECINOS, GEMPP, y CAPRILE, 2005).

Los niños y jóvenes se socializan económica y financieramente en las interacciones con los agentes de socialización como La escuela, amigos, familia y medios de comunicación (HILGERT, HOGARTH y BEVERLY, 2003). La familia, especialmente los padres, constituyen una fuente primaria en la valoración del dinero y el ahorro las tendencias actitudinales hacia el endeudamiento y la comprensión de cómo funciona el mundo 
económico y financiero (CLARKE, HEATON, ISRAELSEN y EGGETT, 2005; (NORVILITIS et al., 2006; LYONS, SCHERPF y ROBERTS, 2006).

En la siguiente figura (Figura 1), podemos observar cómo interactúan los distintos actores en el proceso de socialización económica y cómo ello redunda en su nivel de alfabetización económica (AE) lo que habilita o no al individuo para tomar decisiones.

Figura 1: Modelo de Socialización Económica

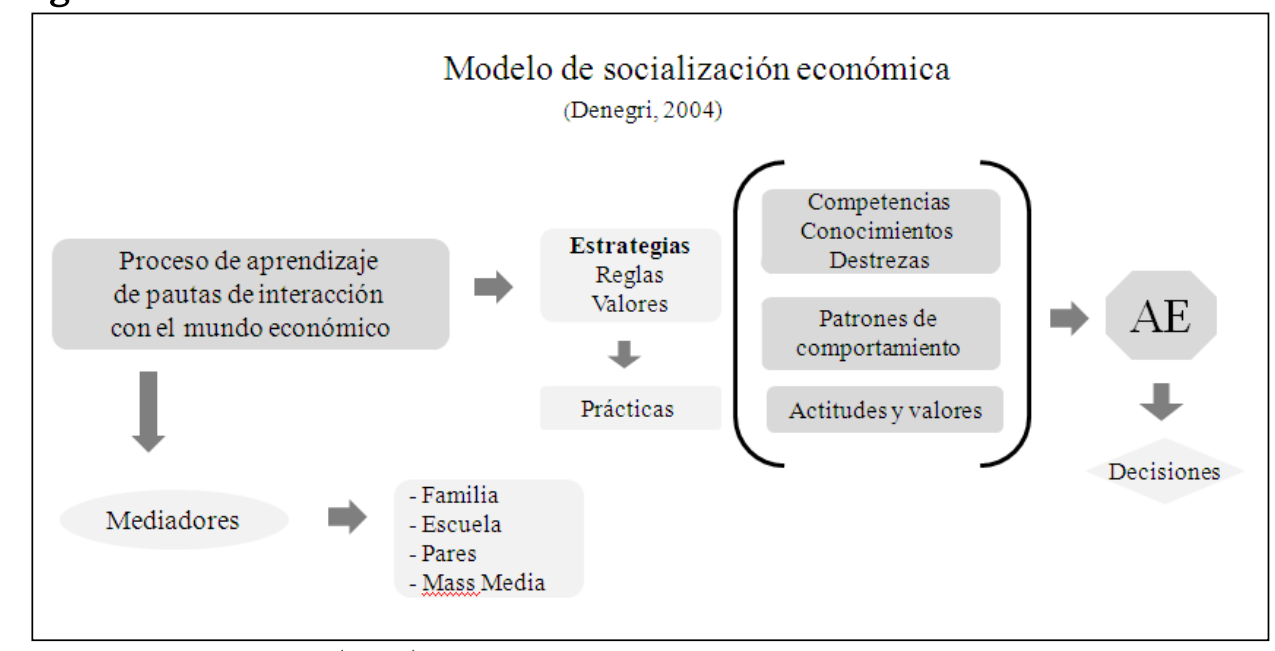

Fuente: Denegri et al. (2005).

En nuestro equipo de trabajo hemos investigado las estrategias y prácticas de socialización económica utilizadas por familias urbanas de diversos niveles socioeconómicos En concordancia con los hallazgos disponibles en la literatura previa (DENEGRI et al, 2005; MORI; LEWIS, 2001), los resultados encontrados indican que la socialización económica comienza tempranamente durante la niñez. Antes de llegar a comprender la complejidad del mundo de la economía los niños han observado, y probablemente imitado, una gran cantidad de estereotipos acerca del consumo, por lo que a un nivel simple han tenido experiencia directa con una amplia variedad de actividades económicas; tal experiencia aumenta durante los años escolares. Sin embargo, también se aprecia que niños y jóvenes adquieren la mayoría de las informaciones y conductas como consumidores de modo informal, ya sea copiando experiencias de sus padres, 
de sus pares y/o por influencia de medios de comunicación de masas, no teniendo acceso a una formación intencionada que les prepare para interactuar con una sociedad de consumo cada vez más agresiva y compleja. Ello es evidente al observar la discordancia entre el discurso de los padres y el de los niños especialmente en cuanto a las prácticas y valores que los adultos dicen fomentar en sus hijos.

Al mismo tiempo, se observa que los padres utilizan como estrategia principal de socialización la "conversación” especialmente valórica y muy cercana a lo que Piaget llamaba "lecciones morales" (PIAGET, 1971; DENEGRI, 2005), donde se repiten varios de los discursos valóricos que los mismos padres internalizaron de sus familias de origen. Si bien la transmisión valórica es un importante elemento de socialización, en el caso de la socialización económica es insuficiente si no va acompañada del desarrollo de competencias para un consumo reflexivo. En el caso de las familias del estudio (DENEGRI et al, 2005), se apreció como los padres dicen "enseñar a comprar" y "ahorrar", sin ser percibida esta enseñanza como tal; por el contrario, a espaldas de los padres los niños comienzan a desarrollar tempranas conductas de endeudamiento informal que pueden marcar futuros estilos de consumo (DENEGRI et al., 2005; DENEGRI; GEMPP; MARTÍNEZ, 2005; DENEGRI et al., 2008). Llama La atención que si bien en el discurso de los padres se reconoce La importancia de educar en el uso del dinero a sus hijos; esto no parece reflejarse coherente mente en las prácticas cotidianas de socialización económica que ellos llevan a cabo. Pese a que los padres dicen valorar un estilo de vida austero, con sus hijos se comportan más bien como consumidores impulsivos, donde la reflexión y los límites a las peticiones de consumo de los niños parecen ser bastante difusas. Por otro lado, si bien valoran la importancia de aprender a administrar el dinero, no fomentan la autonomía ni La responsabilidad efectiva de dicha administración en sus hijos.

En síntesis, los resultados de este conjunto de investigaciones señalan la presencia de estilos diferenciales de socialización económica relacionados 
con el nivel socioeconómico de la familia. En el nivel socioeconómico alto existe un patrón característico de atesoramiento y gasto racional donde las prácticas de socialización económica son más sistemáticas y organizadas. Por su parte, en el nivel socioeconómico medio se observa un patrón consumista a través del escaso ahorro y el gasto no planificado, donde el discurso y las prácticas concretas de socialización generadas por los padres son contradictorias. Por último, en el nivel socioeconómico bajo existe un patrón caracterizado por la supervivencia presentista, los niños consideran destinar gran parte de sus recursos monetarios en necesidades de sus familias, viéndose al dinero más como un instrumento de ayuda familiar por sobre las necesidades personales, sumado a la sensación de incontrolabilidad de la economía individual (LLANOS et al., 2008).

En la misma línea de socialización económica, otras investigaciones han señalado como un factor relevante a la influencia de los pares, siendo esto particularmente fuerte en la adolescencia. Al respecto, se propone que los adolescentes aprenden los aspectos racionales de consumo de sus padres y los aspectos sociales y afectivos relacionados con el materialismo de sus pares (MOORE, 2003), fundamentalmente por la necesidad de pertenencia e identificación, propia del proceso de construcción de identidad adolescente. En esta perspectiva, se interpreta la presión ejercida por los pares como la razón por la que los adolescentes valoran los bienes materiales y las marcas populares moldeando actitudes, valores, normas y aspiraciones de consumo (BATRA et al, 2001) y afectando la elección de productos y marcas (WOOTEN; REED, 2004) e influyendo en el desarrollo, por modelaje, de competencias financieras y económicas (KRETSCHMER; PIKE, 2009, MASCHE， 2010;NEYMOTIN， 2010; OYSERMAN; DESTIN， 2010; FRIEDLINE; ELLIOT; NAM, 2013). Esto sucede en un contexto evolutivo donde los jóvenes están madurando sus destrezas de autocontrol y, por lo tanto, son más susceptibles al poder del mercado (COLLINS; STEINBERG, 2006). 
Sin embargo, no son solo la familia y los pares quienes juegan un rol de mediador en los procesos de socialización y alfabetización económica en la infancia, también es debe relevarse la mediación simbólica ejercida por los medios de comunicación masiva, inicialmente la televisión y más recientemente Internet. Ello constituye una influencia que compite con la familia dada la gran cantidad de tiempo que le dedican los niños cotidianamente (BUKSTEIN, 2007). Durante este tiempo son bombardeados por persuasiones publicitarias de las que no siempre tienen conciencia y fidelizados tempranamente hacia objetos de consumo tanto presentes como futuros. Ello se amplifica si consideramos las características del pensamiento infantil y preadolescente, donde la transición entre la lógica concreta y abstracta, entre lo mágico y lo real, influye profundamente en las condiciones de construcción de identidad (DENEGRI; MARTÍNEZ, 2004), lo cual es aprovechado por las industrias del marketing y los anuncios publicitarios para inducir la identificación del yo con los productos que se consumen (CNTV, 2004; OZGEN, 2003; PAGE; RIDGWAY, 2001). En suma, los medios presentan una doble influencia en la conducta económica infantil; por una parte, constituyen una forma permanente de penetración y persuasión, por ende, de estimuladores de patrones de consumo, y por otra pueden tener un importante rol en el desarrollo de propuestas educativas más motivantes y cercanas a la cultura de los niños (DEL VALLE, 2000; 2003). Sin embargo, lo anterior implica también el requerimiento de desarrollar actividades que permitan "una lectura crítica" de los medios y la preparación de profesores.

\section{Los desafíos de La educación económica y financiera en la formación ciudadana de niños y adolescentes: Una mirada constructivista}

La educación económica y financiera debe estar presente desde muy temprano en la vida de niños y niñas, incorporando la reflexión sistemática sobre problemas económicos reales de la vida cotidiana para ayudarlos a 
diferenciar aquellos aspectos que se consideran claves para comprender el mundo social. Uno de ellos es la comprensión de la diferencia entre concebir a la vida como una secuencia de eventos que suceden fuera de nosotros y donde no tenemos posibilidad de control versus la conceptualización de los fenómenos sociales como resultado de elecciones realizadas por individuos y colectivos. El segundo aspecto clave implica la toma de conciencia que en cada decisión hay implicados costos y beneficios y que ellos deben ser evaluados antes de actuar. Ambos aspectos implican el desarrollo de La capacidad de relacionar procesos, evaluar cursos de acción y reflexionar sobre consecuencias, todo lo cual forma parte importante de las capacidades cognitivas, de comportamiento y actitudinal es necesarias para la instalación de una lógica ciudadana en contraposición con una postura pasiva frente a la realidad social (DENEGRI; MARTÍNEZ, 2004).

Para comprender la complejidad de los procesos que intervienen em el comportamiento económico, es necesario considerar que este no tiene lugar en el vacío o separado de otros aspectos del comportamiento humano, sino que involucra a la persona en su totalidad, con su historia, características personales, sociales y culturales y al contexto general en que ocurre el comportamiento. Por lo tanto, las propuestas de educación financiera deben necesariamente complementarse con la educación ciudadana y con el logro de alfabetización política. Así, los estudios coinciden en la necesidad de un abordaje constructivista que la enlace con problemas de la vida cotidiana que requieran de organización personal y colectiva para ser enfrentados, y especialmente con el aprendizaje de conceptos, el desarrollo de procesos de razonamiento y pensamiento económico y la estimulación de actitudes prosociales que incorporen la alfabetización económica y financiera como parte de las bases de la educación para la ciudadanía (SANTOMERO, 2003; SUITER et al., 2005; LUSARDI; MITCHELL, 2007; LUSARDI; MITCHELL, 2013; THALER; SUNSTEIN, 2008; VAN ROOIJ; LUSARDI; ALESSIE, 2007). 
Por ello, Murphy y Yetmar (2010) señalan que la educación financiera debe estar orientada al cambio de comportamiento y enfatizan la importancia de la toma de decisiones en contextos reales y el enfrentamiento a conflictos cognitivos, ello para que los estudiantes puedan poner efectivamente a prueba sus conocimientos financieros. Otro aspecto clave, es la diferenciación entre los públicos o segmentos hacia los cuales irán dirigidas las estrategias de educación financiera, y allí cobra relevancia considerar que la comprensión del mundo financiero tiene sus particularidades a lo largo del ciclo vital y que ello es especialmente relevante en el caso de los niños y jóvenes, siendo muy importante La consideración del nivel de desarrollo cognitivo y social de los niños y sus propias concepciones en torno al mundo del dinero, la economía y el consumo.

En términos más específicos, la alfabetización financiera es la habilidad de hacer juicios informados y tomar decisiones eficaces para la administración actual y futura del dinero. Incluye la habilidad de comprender las diferentes opciones financieras, planear para el futuro, gastar sabiamente, y saber manejar los retos asociados con las situaciones cotidianas de la vida como la posible pérdida del empleo, el ahorro para el retiro o pagar la educación de los hijos (COHEN; CANDACE, 2011). La alfabetización económica y su complemento financiero se cristalizan conductualmente en el concepto de capacidad financiera, definida como la habilidad y oportunidad de usar el conocimiento y las habilidades implicados en la alfabetización económica y financiera, combinando conocimiento, habilidades y actitudes, con la oportunidad de aplicarlas satisfactoriamente para resolver problemas de la vida cotidiana (COHEN; CANDACE, 2011; LUSARDI; MITCHELL, 2013).

En este contexto, hemos construido una definición de lo que vamos a entender como educación económica, la que se concibe como una acción educativa intencionada cuyo objetivo es aportar a los participantes, las nociones económicas básicas y las estrategias para tomar decisiones 
adecuadas que les permitan situarse ante la sociedad de consumo como personas conscientes, críticas, responsables y solidarias conscientes de sus deberes y responsabilidades como ciudadanos y por lo tanto capacitados para ejercer plenamente su ciudadanía económica.

Estas ideas quedan reflejadas en el modelo de Educación Económica y Financiera (Figura 2) que nuestro equipo ha desarrollado en los últimos años y que se ha validado empíricamente en varias investigaciones.

FIGURA 2: Modelo de Educación Económica y Financiera

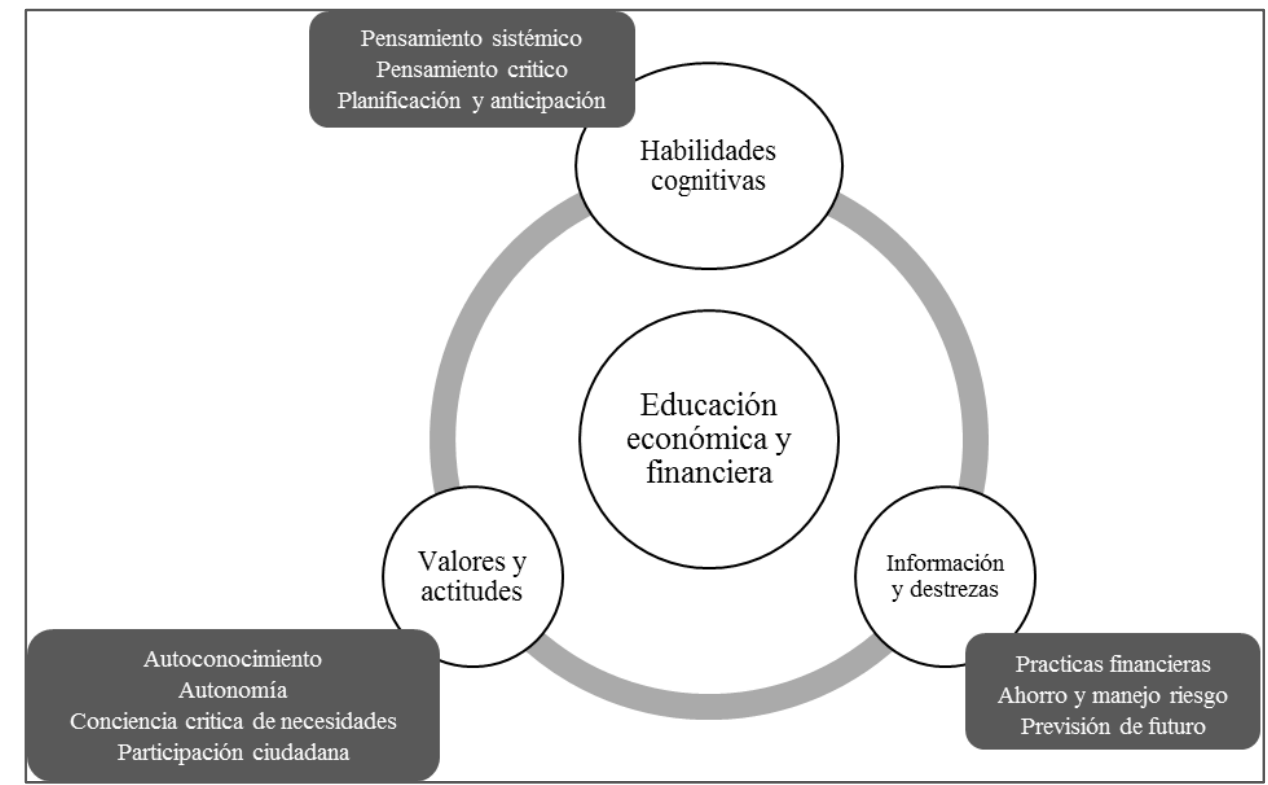

Fuente: Denegri et al., (2014).

Desde una perspectiva constructivista, el modelo articula tres componentes esenciales: 1) Habilidades cognitivas de base; 2) Información y destrezas y 3) Valores y actitudes que son de carácter transversal y cumplen la función tanto de articular los distintos contenidos como aumentar la probabilidad de transferirlos al comportamiento.

Las habilidades cognitivas incluyen el pensamiento sistémico o complejo, pensamiento crítico y capacidad de planificación y anticipación. El pensamiento sistémico se traduce en la habilidad cognitiva de establecer relaciones y complementos y percibir la realidad como un todo. Esta habilidad resulta crucial para comprender los procesos económicos y su 
impacto global. La otra habilidad cognitiva fundamental es el pensamiento crítico, entendido como la capacidad de realizar juicios documentados, discriminar la calidad de la información, exponer correcta y ordenadamente y pensar de manera autónoma, es decir, realizar juicios y tomar decisiones luego del análisis, evaluación y contraste de argumentos, afirmaciones, puntos de vista y evidencias. Habilidad vital para la ciudadanía económica y para el consumo inteligente y sustentable. Finalmente, se incluye la capacidad de planificación y anticipación definida como el proceso mental que permite seleccionar las acciones necesarias para alcanzar una meta, decidir sobre el orden apropiado, asignar a cada tarea recursos necesarios y el establecer el plan de acción adecuado, aspecto vital para la planificación financiera.

El componente de información y destrezas de comportamiento, está constituido por el conjunto de conocimientos y procedimientos de acción que permiten al individuo desarrollar prácticas financieras de uso y administración del dinero eficientes en la vida cotidiana, desarrollar estrategias de ahorro y de manejo del riesgo financiero y planear su futuro financiero previendo el riesgo financiero y desarrollando un plan. En este último punto se incluyen las estrategias de aseguramiento y planeación de ahorros previsionales para un futuro retiro de la vida laboral.

Finalmente, el componente de valores y actitudes es esencial para el desarrollo de una postura crítica ante los impactos del modelo económico en la conducta personal y social y proporciona herramientas para discernir y evaluar el impacto que el comportamiento económico y financiero tiene en la calidad de vida y en el desarrollo personal. Se incluyen aquí el autoconocimiento que permite comprender las motivaciones y emociones a la base de la toma de decisiones económicas, la autonomía que posibilita tomar distancia de las presiones del mercado y publicidad, la conciencia crítica de necesidades que permite diferenciarlas de los deseos y la valoración del ejercicio responsable de la ciudadanía económica. Los componentes interactúan entre si y deben ser abordados en forma transversal e integrada 
para el logro del propósito de una educación financiera crítica y orientada a la formación ciudadana.

Así, este modelo también hace eco de los componentes que el propio Piaget (1986) incorpora como parte de su noción de sociedad y de construcción colectiva de operaciones donde el propio entorno social es concebido como un sistema de actividades cuyas interacciones elementales consisten en acciones que se modifican las unas a las otras de acuerdo con ciertas leyes de organización o de equilibrio y que incorporan acciones técnicas de fabricación y de utilización, acciones económicas de producción y distribución, acciones morales y jurídicas de colaboración o constreñimiento y opresión y acciones intelectuales de comunicación, de búsqueda de información y de crítica mutua.

En cuanto al momento idóneo para incorporar la educación financiera en la infancia, las investigaciones señalan que a partir de los 10 u 11 años están presentes tanto las competencias cognitivas como la autonomía relativa, el interés progresivo en el mundo social $\mathrm{y}$ una mayor susceptibilidad a la influencia del grupo de pares y los medios de comunicación (DENEGRI, 1995b; PIAGET, 1964), este segmento resulta especialmente interesante para focalizar estrategias de educación económica y financiera. El impacto que puede tener una intervención en este grupo etáreo es estratégico dado que por sus características agrupan en si tres tipos de mercado: un mercado presente por la cantidad de recursos que manejan y su autonomía para tomar decisiones de consumo, un mercado futuro dado que en estas edades se establecen actitudes y patrones de consumo que pueden persistir en la edad juvenil y adulta y un mercado de influencia por el impacto que tienen en las decisiones de consumo familiar. Por lo tanto, el logro de perfiles de consumo responsable, eficiente e informado puede impactar no sólo a nivel personal sino también familiar y social.

En cuanto a contenidos, la mayoría de los modelos de educación económica y financiera, coinciden en que es fundamental incorporar la 
relación entre al menos tres variables que interactúan en el comportamiento económico, ellas son: (1) las elecciones económicas, (2) los conceptos económicos y (3) las metas del comportamiento (SCHUG, 1982; 1996; SCHUG; HAGEDORN, 2005). Ello es coincidente, como plantea DONGOMONTOYA (2008) con el sentido del aprendizaje desde la óptica piagetana que implica colocar a los niños y adolescentes en la condición de verdaderos sujetos activos del aprendizaje y por esto en situaciones donde la investigación y el descubrimiento posibiliten una verdadera creación de conocimiento.

La importancia de la experiencia y la actividad en la construcción de conceptos económicos y financieros queda en evidencia en aquellas investigaciones que han mostrado que los niños y los adolescentes que participan activamente en el manejo autónomo de dinero, que deben establecer un presupuesto y que han tenido experiencias de ahorro bancario o de otros productos financieros tienen mayor probabilidad de ahorrar e invertir y ser más cuidosos en sus finanzas personales cuando adultos (JOHNSON; SHERRADEN, 2007; KOTLIKOFF; BERNHEIM, 2001; NATIONAL ENDOWMENT FOR FINANCIAL EDUCATION [NEFE], 2004).

\section{Conclusiones}

La evidencia empírica demuestra que las personas que han estado expuestos tempranamente a procesos de educación financiera desarrollan comportamientos más proclives hacia el ahorro, la planificación de recursos y la previsión de futuro (BERNHEIM; GARRETT; MAKI, 2001; COLE; SAMPSON; ZIA, 2010; LUSARDI, 2009). En esta misma línea, se ha encontrado que mayores niveles de alfabetización financiera se relacionan no solo a la creación de activos, sino también con más capacidades de gestión de la deuda, donde los individuos optan por hipotecas menos costosas, evitando los altos intereses y cargos adicionales (GERARDI; GOETTE; 
MEIER, 2010; LUSARDI; TRUFANO, 2009a; MOORE, 2003). Esta evidencia sugiere una relación causal directa entre educación financiera y cambios en el comportamiento, es decir, un impacto directo en la competencia financiera. Por otra parte, otros estudios señalan una correlación positiva entre el indicador de capital humano evaluado por la prueba PISA y el nivel de alfabetización económica de la población (JAPELLI, 2009), lo que la sitúa como una variable que interviene en el nivel global de desarrollo de un país.

Todas las experiencias internacionales parecen indicar que la educación económica y financiera beneficia a los individuos en todas las etapas de la vida. A los niños les hace comprender el valor del dinero y del ahorro; a los jóvenes les prepara para la vida independiente y a los adultos les ayuda a planificar decisiones básicas como la compra de una vivienda, el mantenimiento de una familia, la financiación de los estudios de sus hijos o a preparar la jubilación.

En términos de beneficios globales, en el plano individual la educación económica y financiera, contribuye a mejorar las condiciones de vida de la población al proporcionar las herramientas necesarias para la toma de decisiones relativas a la planeación para el futuro y a la administración de los recursos, así como información pertinente y clara que da lugar a un mayor y mejor uso de los productos y servicios financieros. Así, los usuarios con mayores niveles de educación financiera tienden a ahorrar más, lo que normalmente se traduce en mayores niveles de inversión y crecimiento de la economía en su conjunto.

A su vez, una ciudadanía educada financieramente contribuye a mejorar el desempeño de las instituciones generando un intercambio de información de mayor calidad entre las instituciones financieras y sus clientes, apoyando la construcción y/o restablecimiento de las confianzas en el sistema financiero, aumentando la competitividad e innovación y mayores mecanismos de autorregulación del propio mercado ante la necesidad de rendir cuentas de su acción frente a una ciudadanía informada y activa. 
Resulta crucial apoyar a los niños y adolescentes para que comprendan las cuestiones financieras y desarrollen competencias, actitudes y valores que les protejan frente a la presión constante del mercado hacia un consumo poco reflexivo y al respecto, la mayoría de los autores coinciden en la importancia que tiene la alfabetización económica y financiera temprana, dado que es en la infancia y adolescencia donde se instalan la mayoría de las actitudes y valores que sustentarán gran parte del comportamiento económico adulto.

Bajo estos principios, surge la interrogante de “¿Qué se debe enseñar en la escuela?, para lo cual la evidencia de la literatura y nuestra propia experiencia en proyectos de aula en educación económica y financiera se orientan a la importancia de habilidades para planificar y gestionar las finanzas personales, la comprensión de los mecanismos de ahorro, endeudamiento e impuestos, la anticipación de riesgos y evaluación de recompensas y aspectos más específicos de los derechos del consumidor y la instalación de actitudes y valores proclives a un uso racional de los recursos y la valoración del ahorro y consumo inteligente (DENEGRI et al., 2014).

Si bien es importante el papel de agente socializador y educador de la familia en el ámbito económico, no es posible ignorar que los propios padres también carecen en muchos casos de una adecuada alfabetización económica y ello se traduce en la transmisión intergeneracional de pautas de consumo y uso del dinero poco reflexivas o ineficientes.

Por ello, se debe intencionar la inclusión curricular temprana de la educación económica y financiera desde la educación básica mediante aproximaciones didácticas que potencien la reflexión, el pensamiento crítico y el trabajo cooperativo para el desarrollo de actitudes y destrezas que permitan a niños y niñas el control y especialmente el autocontrol de sus procesos de consumo. Es evidente que en esta aproximación, el aporte del enfoque constructivista y su conocimiento de las formas sobre cómo se construye el conocimiento tanto en el aula como fuera de ella y las variables que intervienen en este proceso, resulta indispensable para el diseño de 
programas educativos que efectivamente respondan a las necesidades y características de los estudiantes y aporten efectivamente a su formación como ciudadanos críticos y propositivos (PIAGET, 1972).

En conclusión, introducir los temas económicos en el currículo escolar y con ello lograr que nuestros niños y jóvenes sean alfabetizados económicamente, presenta una serie de ventajas específicas. En primer lugar, el desarrollo de las destrezas en el uso y administración del dinero puede ayudar a niños y jóvenes a prepararse de manera real para los problemas de toma de decisiones económicas que deberán enfrentar en la vida adulta, y, por ello, ayuda a la transición entre la vida escolar y laboral. Por otra parte, la alfabetización económica requiere del desarrollo de estrategias de planificación, toma de decisiones y resolución de problemas, las cuales constituyen destrezas transferibles a otros ámbitos de conocimiento y, por tanto, estimulan el desarrollo de capacidades generales de razonamiento.

Finalmente, confrontar a los alumnos con el reconocimiento de su entorno económico y social, de los problemas presentes en él y ayudarlos a construir su juicio crítico en torno a su propia actuación frente al mundo del consumo, estimula el desarrollo de un pensamiento social que constituye la base de la educación para la ciudadanía.

La diferencia entre el ciudadano y el consumidor está dada fundamentalmente porque el primero es capaz de evaluar críticamente el entorno social y asumir el rol que le corresponde como controlador de las decisiones de aquellos a quienes encomendó su representación mediante el voto. Ello involucra el concepto de "accountability" tan necesario para asegurar la gobernabilidad en los sistemas democráticos.

\section{Referências}

AMAR, J.; ABELLO, R.; DENEGRI, M. El desarrollo de conceptos económicos en niños y adolescentes colombianos y su interacción con los sectores educativo y calidad de vida. Informe Final Proyecto COLCIENCIAS, n. 12, 2001. 
Uninorte, 2002.

et al. Desarrollo del pensamiento económico en niños de la región caribe colombiana.Revista latinoamericana de psicologia, Colômbia, v. 35, n. 1, p. 7-18, 2003.

ARAUJO, R. Alfabetização Econômica: compromisso social na educação das crianças. São Bernardo do Campo: Editora Universidade Metodista de São Paulo, 2009.

BATRA, R.; HOMER, P.; KAHLE, L. Values, susceptibility to normative influence, and attribute importance weights: a nomological analysis. Journal of Consumer Psychology, v. 11, n. 2, p. 115-128, 2001.

BAUMANN, Z. Vida de consumo. Buenos Aires: Fondo de Cultura Económica, 2007.

BERNHEIM, D.; GARRETT, D.;MAKI, D. Education and saving: The long-term effects of high school financial curriculum mandates. Journal of Public Economics, n. 85, p. $435-565,2001$.

BERTI, A. E.; BOMBI, A. S. The child's construction of economics. Cambridge: Cambridge University Press, 1988.

; GRIVET, A. The development of economic reasoning in children from 8 to 13 years old: Price Mechanism. Contributi di Psicologia, n. 3, p. 37-47, 1990.

BRAUnSTEIN, S. Director Division of Consumer and Community Affairs, Before the Committee on Financial Services, U.S. House of Representatives. Consultado en:

http://www.federalreserve.gov/newsevents/testimony/ braunstein20080415a.htm, 2008.

BUKSTEIN, M. Relación entre alfabetización económica, prácticas del uso del dinero y hábitos de consumo televisivo en preadolescentes (tweens) de Temuco. Tesis (Magíster en Psicología) - Universidad de La Frontera, Temuco, Chile, 2007.

CLARKE, M. C.; HEATON, M. B.; ISRAELSEN, C. L.; EGGETT, D.L. The acquisition of family financial roles and responsibilities. Family and Consumer Sciences Research Journal, v. 33, p. 321-340, 2005.

COHEN, M.; CANDACE, N. Financial Literacy: a step for clients towards financial inclusion. Microcredit Summit Campaign. Microfinance Opportunities, 2011.

COLE, S.; SAMPSON, T.; ZIA, B. Prices or Knowledge? What Drives Demand for Financial Services in Emerging Markets? HBS Working Papers - forthcoming in The Journal of Finance v. 9, n. 11, 2010. 
COLLINS, W. A.; STEINBERG, L. Adolescent development in interpersonal context. In: W. DAMON, R.; LERNER, W. (Eds.). Social, emotional, and personality development. Handbook of Child Psychology.New York: Wiley, 2006.P. 1003-1067.

CONSEJO NACIONAL DE TELEVISIÓN [CNTV] y MCCANN - ERICSSON. Informe 8/13: Los tweens Chilenos. Maval. Santiago, 2003.

CONSEJO NACIONAL DE TELEVISIÓN [CNTV]. Zoom Tweens. Estudios Cualitativos. Santiago de Chile. CNTV, 2004.

DEL VALLE, C. La producción de comunicación (medial) en contextos educativos interculturales. Revista Educación y Humanidades, v. 9, p. 27-37, 2000.

La comunicación mediática. Problemas epistemológicos y pragmáticos en la cobertura y tratamiento de contenidos de interés social. Tercer Milenio - Revista de Comunicación y Periodismo, v. 8, p. 18-20, 2003.

DENEGRI, M. El desarrollo de las ideas acerca de la emisión monetaria en niños y adolescentes: estudio exploratorio. Revista del Instituto de Ciencias de la Educación, n. 9, enero-abril, p. 47-62,1995a.

El desarrollo de las ideas acerca del origen y circulación del dinero: un estudio evolutivo con niños y adolescentes. Ediciones Universidad Autónoma de Madrid. España, 1995b.

Psicogénesis de las ideas en torno a la relación Estado-economía. In: GUZMÁN,L. (Ed.) Exploraciones en Psicología Política. Universidad Diego Portales: Santiago, 1997.

Proyectos de aula interdisciplinarios y reprofesionalización de profesores: un modelo de capacitación. Estudios Pedagógicos, v. 31, n. 1, p. 35-50, 2005.

et al. Desarrollo del pensamiento económico en la infancia y adolescencia. Boletín de Investigación Educacional, v. 13, p. 291-308, 1998.

et al. Informe Final Proyecto Fondecyt, n. 1970364.Fondo Nacional de Ciencia y Tecnología. Santiago, Chile, 2000.

et al. Socialização econômica em famílias chilenas de classe média: educando cidadãos ou consumidores? Psicologia\&Sociedade, Belo Horizonte, v. 17, n. 2, p. 8898, 2005.

et al. Prácticas de ahorro y uso del dinero en pre adolescentes (tweens) chilenos.Revista UNIVERSUM, v. 23, n. 1, p. 24-38, 2008.

et al. Educación Económica y Financiera para la formación inicial de profesores. In: SEPÚLVEDA, J. (Ed.). Herramientas conceptuales y didácticas. Chile: Ediciones Universidad de La Frontera, 2014. 
.; GEMPP, R.; MARTÍNEZ, G. Estrategias de Socialización Económica en Familias de Clase Alta y Media- Alta y su impacto en las prácticas de uso del dinero de los hijos. Boletín de Investigación Educacional, v. 20, n. 2, p. 41 -60, 2005.

;MARTÍNEZ, G. ¿Ciudadanos o consumidores? Aportes constructivista a la educación para el consumo. PAIDEIA - Revista de Educación, Ribeirão Preto, v. 37, p. 101-116, 2004.

.; ___ _ETCHEBARNE, S. La comprensión del funcionamiento bancario en adolescentes chilenos: un estudio de psicología económica. Interdisciplinaria, v. 24, n. 2, p. 137-160, 2007.

DONGO-MONTOYA, A. La teoría del aprendizaje de Piaget y sus consecuencias para la praxis educativa. Revista IIPSI, v. 11, n. 1, p. 167- 181, 2008.

FRIEDLINE, T.; ELLIOTT, W. Connections with banking institutions and diverse asset portfolios in young adulthood: children as potential future investors. Children and Youth Services Review, v. 35, n. 6, p. 994-1006, 2013.

FURTH, H.G. The world of grown - ups. Children 's conceptions of society. Nueva York: Elsevier North Holland, 1980.

GEMPP, R. et al. Medición de la alfabetización económica en niños: una aplicación del modelo de crédito parcial.Revista Psykhe, v. 15, n. 1, p. 13-28, 2006.

GERARDI, K.; GOETTE L.; MEIER, S. Financial literacy and subprime mortgage delinquency: evidence from a survey matched to administrative data. Working Paper - Federal Reserve Bank of Atlanta, April, 2010.Disponívelem:https://www.frbatlanta.org//media/documents/research/publications/wp/2010/wp1010.pdf

HILGERT, M. A.; HOGARTH, J. M.; BEVERLY, S. G. Household Financial Management: The Connection between Knowledge and Behavior. Federal Reserve Bulletin, v. 89, n. 7, p. 309-322, 2003.

JAHODA, G. European lag in the development of an economic concept: a study in Zimbabwe. British Journal of Developmental Psychology, n. 1, p. 113-120, 1983.

JAHODA, G. The development of thinking about socio-economic systems. In: TAJFEL, H. (Ed.). The social dimension. Vol. I, Cambridge: Cambridge University Press, 1984.

JAPELLI, T. Economic Literacy: an International comparison. Centre for studies in economics and finance, University of Salerno, n. 238, 2009.

JOHNSON, M; SHERRADEN, M. From Financial Literacy to Financial capability Among Youth. The Journal of Sociology \& Social Welfare, v. 34, n. 3, 2007.

KHAN, S.; NARU, A. Modernizing economic education in the global economy with reference to Pakistan. Journal of Asian Economics, v. 17, n. 5, p. 904-922, 2006. 
KRETSCHMER, T; PIKE, A. Links between nonshared friendship experiences and adolescent siblings' differences in aspirations. Journal of Adolescence, v. 33, n. 1, p. 101-10, 2009.

KOTLIKOFF, L. J.; BERNHEIM, B.D. Household financial planning and financial literacy. In: KOTLIKOFF, L. J. (Ed.) Essays on saving, bequests, altruism, and lifecycle planning. Cambridge, MA: MIT Press, 2001. p. 427-478.

LUSARDI, A. U.S. Household savings behavior: the role of financial literacy, information and financial education programs .In: FOOTE, C.; GOETTE, L.; MEIER, L. (Eds.). Policymaking Insights from Behavioral Economics. Boston: Federal Reserve Bank of Boston, 2009. p.109-149.

.; MITCHELL, O. Baby boomer retirement security: the roles of planning, financial literacy, and housing wealth. Journal of Monetary Economics, v. 54, n. 1, p. 205-224, 2007.

.; The economic importance of financial literacy. Theory and evidence: National Bureau of Economic Research, 2013.

.; TRUFANO, P. Debt literacy, financial experiences, and over indebtedness. NBER Working Paper, n. 14808, 2009.

LYONS, A.A profile of financially at-risk college students. The Journal of Consumer Affairs, v. 38, p. 56-80, 2004.

SCHERPF, E.; ROBERTS, H. Financial Education and Communication between Parents and Children. Journal of Consumer Education, v. 23, p. 64-76, 2006.

LLANOS, M. et al. Aprendiendo a comprender el mundo económico. Ediciones Uninorte. Colombia, 2008.

MASHE, J.G. Explanation of normative declines in parents' knowledge about their adolescent children. Journal of Adolescence, v. 33, n. 2, p. 271-284, 2010.

MORI, E.; LEWIS, A. Money in the contemporary family.Nestle Family Monitor, v. 20, n. 3, p. $21,2001$.

MOORE, D. Survey of financial literacy in Washington State: knowledge, behavior, attitudes, and experiences. Washington State University: Social and Economic Sciences Research Center, 2003.

MURPHY, D.; YETMAR, S. Personal financial planning attitudes: A preliminary study of graduate students. Management Research Review, v. 33, n. 8, p. 811-817, 2010 .

NATIONAL ENDOWMENT FOR FINANCIAL EDUCATION (NEFE). What is the NEFE high school financial planning program? 2004. Disponível em: http://www.nefe.org/ 
hsfppportal/includes/main/home.asp?portal=4\&page=4000\#evaluation2, 2004.Acesso em: 03 de Abril de 2006.

NEYMOTIN, F. Linking self-esteem with the tendency to engage in financial planning. Journal of Economic Psychology, v. 31, n. 6, p. 996-1007, 2010.

NORVILITIS, J.; SANTA MARÍA, P. Credit card debt on college campuses: causes, consequences, and solutions. College Student Journal, v. 36, p. 356-364, 2002.

; et al. Personality Factors, Money Attitudes, Financial Knowledge, and Credit-Card Debt in College Students. Journal of Applied Social Psychology, v. 36, p. 1395-1413, 2006.

.; SAZBLICKI, J.; WILSON, S. Factors Influencing Levels of Credit-Card Debt in College Students. Journal of Applied Social Psychology, v. 33, n.5, p. 935947, 2003.

ORGANISATION FOR ECONOMIC CO-OPERATION AND DEVELOPMENT [OECD] Improving financial literacy: analysis of issues and policies. OECD Publishing, 2005a.

. Recommendation on Principles and Good Practices for Financial Education and Awareness. OECD Publishing, Directorate for Financial and Enterprise Affairs, 2005b.

Improving Financial Education and Awareness on Insurance and Private Pensions. OECD Publishing, 2008.

. Financial literacy and consumer protection: overlooked aspects of the crisis. 2009. Disponível em: http://www.financialeducation.org/dataoecd/32/3/43138294.pdf

.Closing the Gender Gap: Act now. OECD Publishing, 2012.

PISA 2012 Results - Students and Money: Financial Literacy Skills for the 21st Century. V. IV. PISA: OECD Publishing, 2014.

OYSERMAN, D.; DESTIN, M. Identity-based motivation: Implications for intervention. The Counseling PSychologist,v. 38, p. 1001-1043, 2010.

OZGEN, O. An analysis of child consumers in Turkey. International Journal of Consumer Studies, v. 27, p. 366-380, 2003.

PAGE, C.; RIDGWAY, N. The impact consumer environments on consumption patterns on children from disparate socioeconomic backgrounds. Journal of Consumer Marketing, v. 18, p. 21-40, 2001.

PIAGET, J. El criterio moral en el niño. Barcelona: Fontanella, 1971. 
Desarrollo y aprendizaje.In: Piaget rediscovered: a report of the Conference on cognitive studies and curriculum development. Ithaca: Cornell Univ. Press, 1964. p. 7-20.

.Fondements scientifiques pour l'éducation de demain.Perspectives: revue trimestrielle de l'éducation, París, v. 2, n. 1, p. 13-30, 1972.

. Estudios Sociológicos. Editorial: Planeta Agostini, Barcelona, 1986.

PROGRAMA DE LAS NACIONES UNIDAS PARA EL DESARROLLO [PNUD]. Desarrollo humano en Chile: la manera de hacer las cosas. Chile: PNUD, 2009.

SALEMI, M. K. Teaching economic literacy: Why, what and how. International Review of Economics Education, v. 4, p. 46-57, 2005.

SANTOMERO, A. Knowledge is power: the importance of economic education. Business Review, v. 5, n. 1, 2003.

SCHUG, M. Economic education across the curriculum. Bloomington: Phi Delta Kappa Educational Foundation, 1982.

SCHUG, M.; HAGEDORN, E. The money savvy pig tm goes to the big city: testing the effectiveness of an economics curriculum four young children. The Social Studies, v. 96, n. 2, p. 68-71, 2005.

SUITER, M. et al. The math factory. In: GRENIER, M. P. (Ed), Mathematics \& economics: connections for life, grades 3-5. New York: National Council on Economic Education, 2005.p. 57-68.

THALER, R.; SUNSTEIN, C. Nudge: improving decisions about health, wealth, and happiness. New Haven: Yale University Press, 2008.

WOOTEN, D.; REED, A. Playing it safe: susceptibility to normative influence and protective self-presentation. Journal of consumer research, v. 31, n. 3, p. 551-556, 2004.

VAN ROOIJ, M.; LUSARDI.A; ALESSIE, R. Financial literacy and stock market participation. Documento de Trabajo. Ann Arbor, MI: University of Michigan Retirement Research Center, 2007. Disponível em: http://www.mrrc.isr.umich.edu/publications/papers/pdf/wp162.pdf

ZMUDA, N. Coca Cola launches global music effort to connect with teens advertisingage. Disponível em: http://adage.com/article/global-news/coca-colalaunches-global-music-effort-connect-teens/149204/ 